\title{
The Influence of a Halo on the Evolution of Elliptical Configurations
}

\author{
F.THEEDE and K.O.THIELHEIM \\ Institut für Reine und Angewandte Kernphysik, Abt. Mathematische Physik \\ Christian-Albrechts-Universität zu Kiel, Otto-Hahn-Platz 3, Germany
}

\begin{abstract}
Results of n-body simulations of self-gravitating systems containing 100,000 particles are presented. A program based on the particle-mesh method is used to compute the evolution of a hot stellar-disk till an oval configuration at the center is formed. The system is evolved again from this point onwards a) with halo, b) without halo, and c) with the halo but with total mass of the system reduced to that of case b. Results are presented in form of a diagram showing the development of ellipticity.
\end{abstract}

\section{The Program}

The program is based on a two-dimensional particle-mesh method in a polar grid $(r, \phi)$ with exponentially increasing radial coordinates (Miller 1976, Sellwood 1981):

$$
r=l e^{\alpha u} \quad, \phi=\alpha v \quad, \text { and } \quad \alpha=\frac{2 \pi}{v_{\max }} .
$$

It has been vectorized for the CRAY XMP in Kiel. For calculating the potential we are using a FFT method. The softening parameter is chosen to be $s=1.2 k p c$.

The parameters of the grid are :

$$
l=0.2856 k p c \quad, \quad v_{\max }=64 \text {, and } u_{\max }=60 \text {. }
$$

\section{The Initial Configuration}

The simulation is started with 100,000 stars of total mass $M=10^{11} M_{\odot}$ in a homogeneous disk of radius $10 k p c$ where $M_{\odot}$ is the solar mass.

The angular velocity of a star is given by

$$
\omega(r)=\kappa \sqrt{\frac{G M_{r}}{r^{3}}},
$$

where $0 \leq \kappa \leq 1$ is the relative reduced initial angular momentum (Thielheim 1990), $G$ the gravitational constant and $M_{r}$ the integral mass inside the radius $r$. The initial velocity dispersion is chosen to be isotropic-gaussian, with

$$
\left|\frac{2 K}{W}\right|=0.0025
$$

where $W$ is the total potential energy and $K$ the total kinetic energy.

\section{The Simulations}

For defining an ellipse and calculating the ellipticity a method based on determining the diagonalized tensor of inertia $\left(I_{k l}\right)$ is used. The ellipticity in this case is defined as

$$
e=\max \left\{\begin{array}{lll}
1-\sqrt{I_{11} / I_{22}} & : & I_{11}<I_{22} \\
1-\sqrt{I_{22} / I_{11}} & : & I_{22}<I_{11}
\end{array}\right.
$$




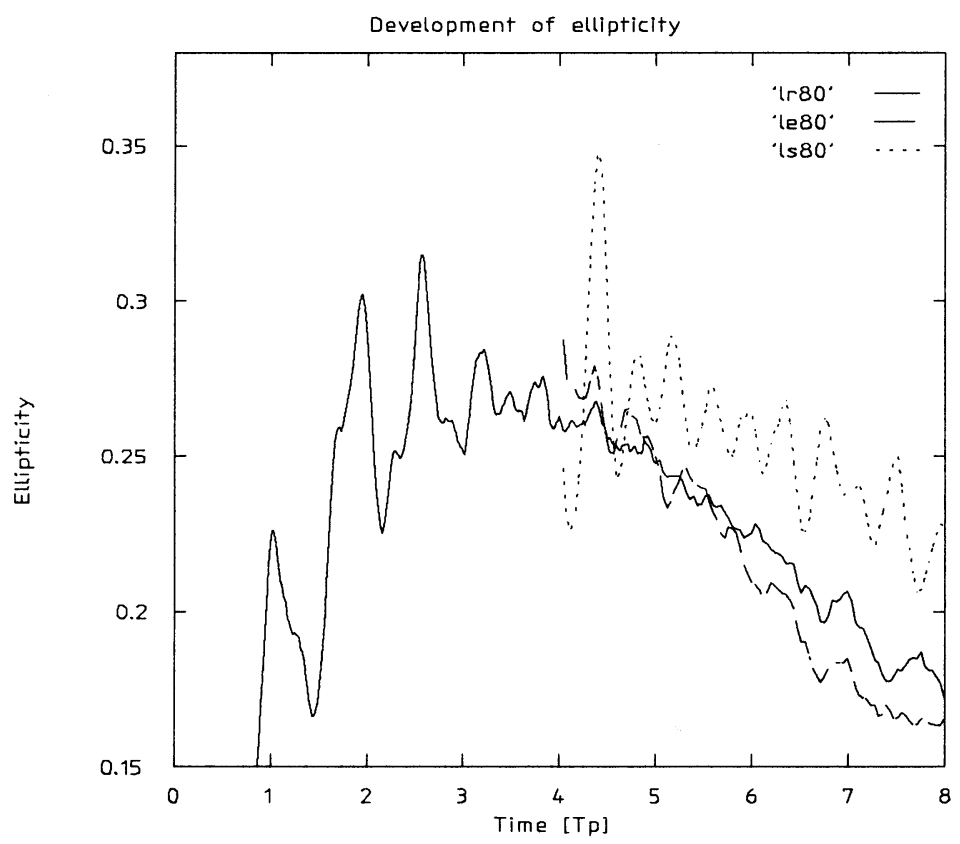

Fig. 1: Development of ellipticity

At the time $4 T_{p}$ the elliptical configuration formed in $\operatorname{lr} 80$ is cut out and simulated as $l e 80$ till $8 T_{p}$ both shown in fig. 1 . If the corresponding number of stars is taken away at random from $\operatorname{lr} 80$ at $4 T_{p}$ the subsequent evolution of $l s 80$ exhibits a development of ellipticity which is also shown in fig. 1.

Comparing these results it is obvious that the influence of the halo on the evolution of the system is modest.

\section{References}

Miller, R.H., 1976, J.Comp.Phys., 21, 400

Sellwood, J., 1981, Astron. Astrophys., 99, 362

Thielheim, K.O., 1990, Astrophys. Space Sci., 170, 381 